\title{
Deep Levels in InGaN/GaN-LEDs
}

\author{
Nazir A. Naz ${ }^{1, *}$, M. Imran ${ }^{2}$, Nabeela Akbar ${ }^{3}$, Akbar Ali $^{1,2}$ \\ ${ }^{1}$ Department of applied Physics, Federal Urdu University of Arts, Science and Technology, Pakistan \\ ${ }^{2}$ Department of Basic Sciences, Riphah International University, Pakistan \\ ${ }^{3}$ Department of Physics, COMSATS Institute of Information Technology, Pakistan
}

Copyright (C) 2015 by authors, all rights reserved. Authors agree that this article remains permanently open access under the terms of the Creative Commons Attribution License 4.0 International License

\begin{abstract}
Study of defects in a semiconducting material can help in improving the electrical and optical properties of a device based on such material. There is a general paucity of knowledge about the nature and origin of deep level defects in ternary and quaternary semiconductors in literature. It is, therefore, of interest to study defects in ternary semiconductor, InGaN-based, LEDs. By employing deep level transient spectroscopy (DLTS), at least nine defects, labeled, E1 - E9 have been observed in InGaN-LEDs. Of these, seven defects E2 - E8 have been characterized. Respective energy states induced by the defects within the band gap are found to be $0.61,1.00,1.24,1.37,1.46,1.68$, $2.25 \mathrm{eV}$ and capture cross-sections, at infinite temperature, are $\quad 2.27 \times 10^{-17}, 4.39 \times 10^{-29}, \quad 1.37 \times 10^{-20}, \quad 9.58 \times 10^{-22}$, $4.61 \times 10^{-28}, 2.19 \times 10^{-24}$ and $8.23 \times 10^{-22} \mathrm{~cm}^{2}$. Concentrations of the defects were estimated to be $4.6 \times 10^{4}, 2.7 \times 10^{4}, 19.2 \times 10^{4}$, $6.9 \times 10^{4}, \quad 6.9 \times 10^{4}, \quad 5.9 \times 10^{4}$ and $6.3 \times 10^{4} \quad\left(\mathrm{~cm}^{3}\right)$, respectively.
\end{abstract}

Keywords InGaN, LEDs, MOCVD, Defects, DLTS

\section{Introduction}

Multi quantum wells (MQWs) in active layer of InGaN have played a pivoted role in the development of light emitting diodes (LEDs), laser diodes (LDs) and solar cells [1-3]. The way, electrons and holes recombine within active layer is of significant importance. They recombine either radiatively to emit photons or non radiatively causing unwanted heat and loss of input energy [3-6]. Non radiative recombination has adverse effects on the efficiency of electronic devices [5]. Usually, deep energy states caused by defects are responsible for radiative and non radiative recombination; therefore, study of defects is of great importance to enhance the luminescent efficiency. Among the other characterization techniques, deep level transient spectroscopy (DLTS) [7] is a powerful technique for obtaining knowledge about the characteristics of such defects.
In earlier studies, Dyba et al. [8] have detected two deep level traps in their metal-organic vapor-phase epitaxial (MOVPE)-grown GaN p-n junctions, while Tsarova et al. [9] have observed four deep level defects in GaN-based laser structure grown by molecular-beam epitaxy (MBE). By employing DLTS, we report here nine deep levels in metal-organic chemical-vapor deposition (MOCVD)-grown InGaN PIN structure.

\section{Experimental}

Green emitting high bright LEDs were imported from Hebei I.T, China [10]. The LEDs emit single green color. InGaN, ternary alloy, grown by low pressure metal organic chemical vapor deposition (LP-MOCVD), lattice matched to $\mathrm{GaN}$, was used to fabricate LEDs. Maximum current at 2 volts reverse voltage is $1.5 \mu \mathrm{A}$ at $25{ }^{\circ} \mathrm{C}$ ambient temperature. The dominant wavelength emitted by the samples is $\sim 525$ nm. Capacitance-Voltage characteristics of LEDs were measured to check the quality of diodes, using capacitance meter, Boonton 7200. In order to check the nature of the junction $1 / \mathrm{C}^{2}$ and $1 / \mathrm{C}^{3}$ were plotted against reverse voltage. The data points in plot, $1 / \mathrm{C}^{2}$ verses applied reverse voltage $\mathrm{V}$, are found approximately fit to a straight line, indicating junction as step junction [11]. However, a careful examination shows that two different straight lines can be fit in the voltage range $0-4.0$ and $7-10$ volts, which indicate that shallow level concentration on both $\mathrm{p}$-and $\mathrm{n}$ - sides of the junction is nearly equal [9].

Deep level spectroscopy, DLS-83D was used to study deep levels in the material. This spectrometer provides a quiescent reverse bias and filling pulse with a repetition rate from $0.1 \mathrm{~Hz}$ to $1 \mathrm{KHz}$ and temperature accuracy of better than $1 \mathrm{~K}$. Outputs of the temperature sensor and Spectrometer were fed to C.P.U through a data bus. Shielded cables were used to provide connections from the samples to the spectrometer. Samples were mounted in a sample holder of the cryostat and electrical connections were provided with the help of two spring-loaded pins. Temperature of the sample is lowered by slowly lowering the sample holder into liquid nitrogen container and raised to room temperature or 
above, by gradually lifting the sample holder or increasing the current through a heater embedded inside the sample holder. The emission of charge carriers from a deep level changes the capacitance of the diode under quiescent reverse bias, which is monitored by spectrometer. Output of the spectrometer, plotted against temperature, gives a DLTS scan. For maximum emission rate of a defect, a peak appears in the scan at a fixed temperature. DLTS scan exhibits different peaks for different defects. Temperature scans were recorded using reverse bias of 3.0 $\mathrm{V}$ and filling pulse of $500 \mathrm{~Hz}$. The DLTS temperature scans were obtained at various emission-rates to estimate the energy-position of the deep levels. From the slope of the best fit line of $\ln \left[\mathrm{e}_{\mathrm{n}} / \mathrm{T}^{2}\right] \mathrm{Vs}$ $1000 / T$ plots, thermal activation energy of defects [7] was estimated. The y-intercept of $\ln \left[\mathrm{e}_{\mathrm{n}} / \mathrm{T}^{2}\right] \mathrm{Vs} 1000 / \mathrm{T}$ plots gives apparent capture cross section $\left(\sigma_{\infty}\right)$ for the deep level defects.

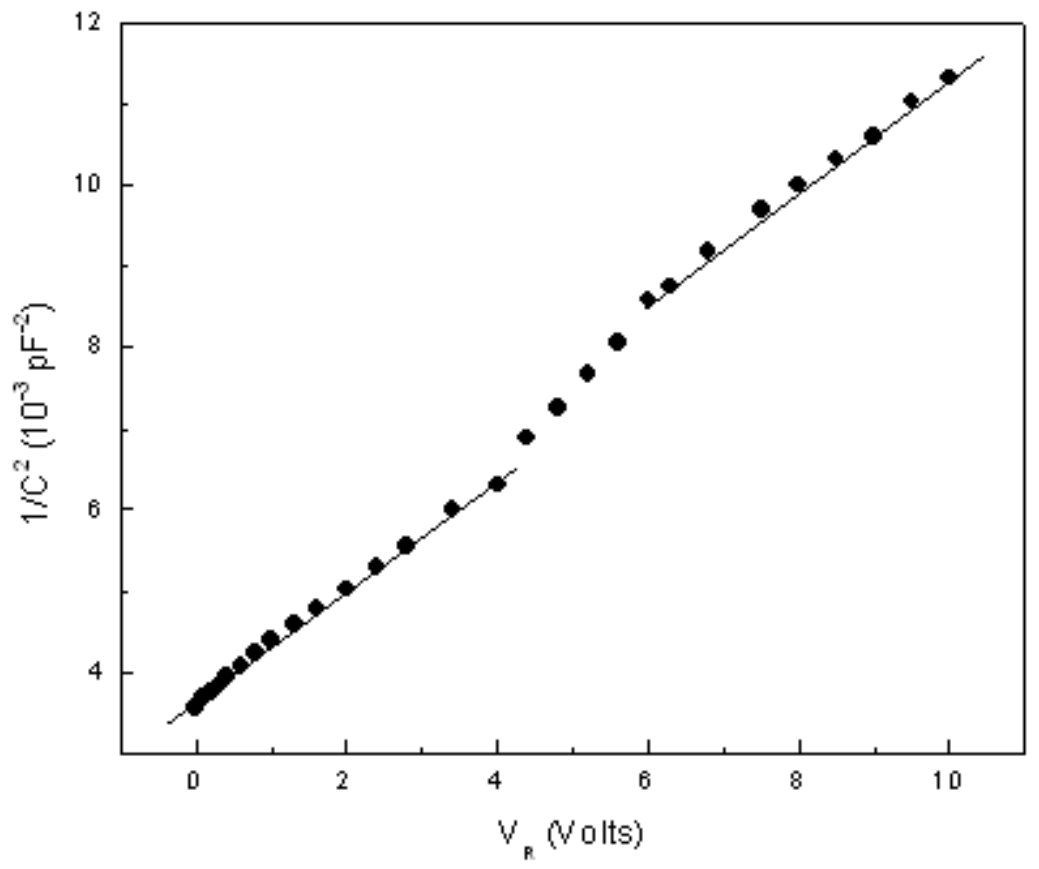

Figure 1. $1 / \mathrm{C}^{2}$ versus reverse voltage plot of InGaN-LEDs.

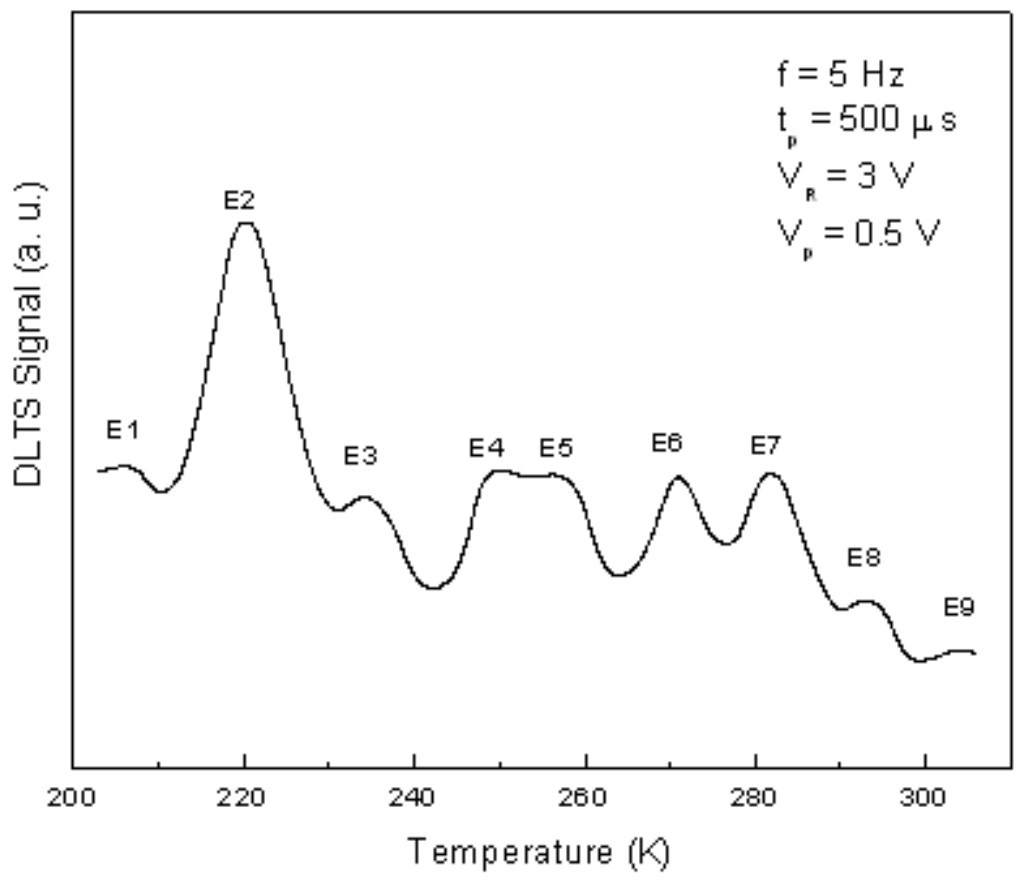

Figure 2. A typical DLTS temperature scan obtained at $5 \mathrm{~Hz}$. 


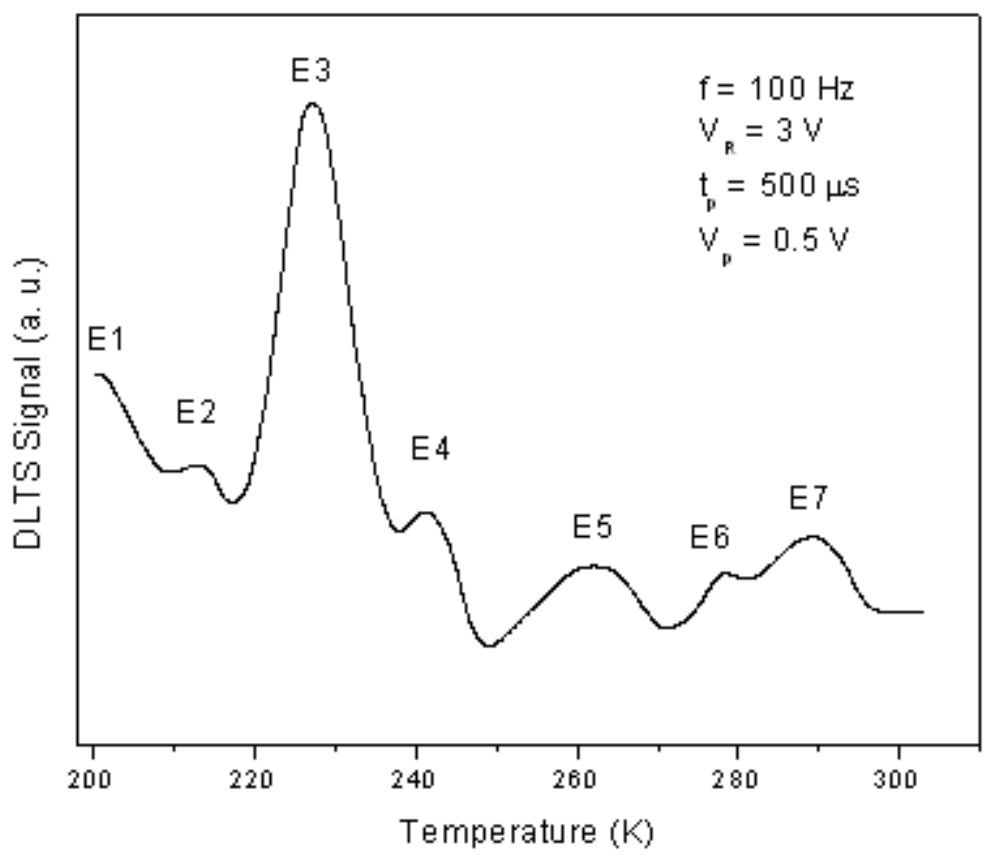

Figure 3. A typical DLTS temperature spectrum taken at $100 \mathrm{~Hz}$.

\section{Results and Discussion}

Typical DLTS scans taken at $5 \mathrm{~Hz}$ and $100 \mathrm{~Hz}$ are shown in Figs. 2 and 3, respectively. Overall nine peaks labeled, E1-E9 have been detected in both DLTS spectra but only seven peaks E2 - E8 could be studied here. Peaks E1 and E9 could not be characterized at this stage of work due to their positions at lower and higher temperatures, respectively. Repetition rates and corresponding temperature range limit the data points for obtaining meaningful results. To calculate thermal activation energies $\left(\mathrm{E}_{\mathrm{A}}\right)$ and capture cross-sections $\left(\sigma_{n}\right)$ for the defects E2-E8, the emission rate data for the deep levels is plotted on semi-log scale, one such plot corresponding to deep level E6 is shown in Fig.4. The activation energies $\left(E_{A}\right)$ and capture cross-sections $\left(\sigma_{n}\right)$ of defects, E2-E8 are given in Table 1.

Table 1. Activation energies and, Capture cross-sections of the defects.

\begin{tabular}{ccc}
\hline Peak & Activation Energy $(\mathrm{eV})$ & $\begin{array}{c}\text { Capture Cross-section }\left(\sigma_{\mathrm{n}}\right) \\
\mathrm{cm}^{2}\end{array}$ \\
\hline & & \\
E2 & 0.61 & $2.27 \times 10^{-17}$ \\
E3 & 1.00 & $4.39 \times 10^{-29}$ \\
E4 & 1.24 & $1.37 \times 10^{-20}$ \\
E5 & 1.37 & $9.58 \times 10^{-22}$ \\
E6 & 1.46 & $4.6 \times 10^{-28}$ \\
E7 & 1.68 & $2.19 \times 10^{-24}$ \\
E8 & 2.25 & $8.23 \times 10^{-22}$ \\
& & \\
\hline
\end{tabular}

The present study highlights a number of defects in InGaN material introduced during growth. In the design of a LED, many layers play their role, therefore; interface of every layer may become a source of defects [12]. These layers may be named as buffer layer, cladding layer, active layer and Tensile Strain Barrier Reducing layer (TSBR) [13]. Though TSBR layer mediate layer to layer lattice mismatch [14] but cannot reduce defects generated at the interface. We compare our data with the published data on $\mathrm{GaN}$ and related IIInitrides materials [14-16]. It was found that the energy sate appearing around $0.61 \mathrm{eV}$ in our work may be the same energy state reported by other workers [16]. The defect was attributed either to single vacancy or $\mathrm{V}_{\mathrm{Ga}}-\mathrm{O}_{\mathrm{N}}$ complex [16]. The other defects could not be identified with any of previously published defects [14-16]. Most likely, the deep levels, E3-E8 detected in our work are related either to background defects or defects generated at the interface. A more detailed work is in progress for further analysis of data and identification of the defects.

\section{Conclusions}

Present DLTS study of deep levels in InGaN/GaN LEDs grown by LP-MOCVD leads to the following conclusion. Overall 09 deep level defects have been detected in InGaN samples. Of these only seven defects could be studied. The defect around $0.61 \mathrm{eV}$ is most likely related either to single vacancy or $\mathrm{V}_{\mathrm{Ga}}-\mathrm{O}_{\mathrm{N}}$ complex. Most probably the other defects originate due to the interface of each layer. 


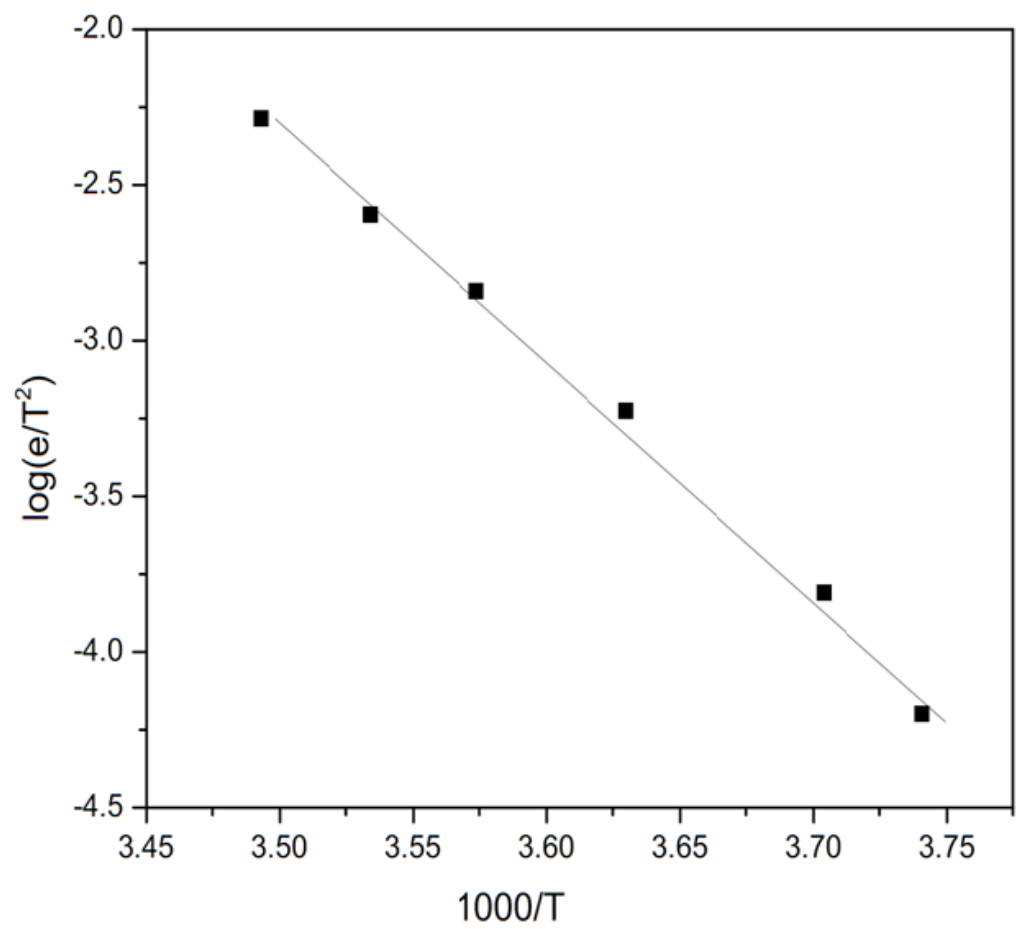

Figure 4. A typical semi-log plot for the defect E6. Solid points are the data points, while line through these points is the least square fitting to data.

\section{REFERENCES}

[1] Zhe Chuan, Feng, Jeng-Hung Chen,Alan Gang Li and L. C Chen: Journal of Physics Conference Series, Vol. 28, 42-47 (2006).

[2] S. Nakamura, S. pearton and G. Fasol, The Blue Laser Diode, springer, Berlin, 2000, pp 1-13, DOI, 10.1007/978-3-660-04156-7.

[3] Mu-TauChu, When-Yih Liao, Ray-Hua Horng, Tsung-yen Tsai, Tsai-Bau $\mathrm{Wu}$, Shu-Ping Liu, Ming-Hsien $\mathrm{Wu}$, and Ray-Ming Lin, IEEE, Elecron Device Letter, Vol. 32, 922-924 (2011).

[4] Manel Charfeddine, Malek Gassoumi, Hana Mosbahi, Christophe Gaquiére, Mohamed Ali Zaidi, Hassen Maaref, Journal of Modern Physics, Vol. 2, 1229-1234 (2011).

[5] Ya-Ju Lee, Chia-Jung Lee, Chih-Hao Chen, Jpn. Journal of Applied Physics, Vol. 50, 04DG 18, 1-5 (2011).

[6] K. Sugiura, K. Domen, M. Sugawara, C. Anayama, M. Kondo, T. Tanahashi, K. Nakajima, Journal of Applied Physics, 70 (9), 4946 - 4949 (1991)
[7] D. V. Lang, Journal of Applied Physics, Vol. 58, 3023 (1974).

[8] P. Dyba, E. Placzek-Popko, E. Zielony, Z. Gumienny, S. Grzanka, R. Czernecki and T. Suski, Acta Physica Polonica A, Vol. 119, 669 (2011).

[9] T. Tsarova, T. Wosinski, A. M. kosa, C. Skierbiszewski, I. Grzegory and P. Perlin, Acta Physica Polonica A, Vol. 112, 331 (2007).

[10] Data sheet Hebei International Trading, Co. Ltd. China, www.hebeiltd.com.cn.

[11] Donald Neamen, Semiconductor Physics and Devices, published by University of Mexico.

[12] Byungjin, M. A. Soohaeng, Cho Changyun Lee, Sangbum Lee, Joonseok Kang, Bumjoon Kim, Donghoon Kang, Youngchul Shin, Youngmin Kim, Taegeum Kim2, YongjoPark, Jpn. Journal of Applied Physics,Vol.45 No. 2A, 774-777 (2006).

[13] Juh-YuhSu,Hsin-ChuanWang, Wen-BinChen, Shi-MingChen, Meng-ChyiWu, Hao - Hui Chen andYan-KuinSu, IEEE Transection Seon Electron devices, Vol. 50, 12 (2003).

[14] K. Sugra, JAP, Vol. 70 (9), 4946 - 4949 (1991).

[15] T. G. Kima, PhysicaB, 376, 610-613 (2006).

[16] M. Asghar, P. Muret, B. Beaumont, P. Gibart, Mater. Sci. Eng. B, Vol. 113, 248 (2004). 